\title{
Fixed point results for contractions involving generalized altering distances in ordered metric spaces
}

\author{
Hemant Kumar Nashine ${ }^{1}$, Bessem Samet ${ }^{2}$ and Jong Kyu Kim ${ }^{3 *}$
}

* Correspondence: jongkyuk@kyungnam.ac.kr ${ }^{3}$ Department of Mathematics, Kyungnam University, Masan, Kyungnam 631-701, Korea Full list of author information is available at the end of the article

\begin{abstract}
In this article, we establish coincidence point and common fixed point theorems for mappings satisfying a contractive inequality which involves two generalized altering distance functions in ordered complete metric spaces. As application, we study the existence of a common solution to a system of integral equations.

2000 Mathematics subject classification. Primary $47 \mathrm{H} 10$, Secondary $54 \mathrm{H} 25$

Keywords: Coincidence point, Common fixed point, Complete metric space, Generalized altering distance function, Weakly contractive condition, Weakly increasing, Partially ordered set
\end{abstract}

\section{Introduction and Preliminaries}

There are a lot of generalizations of the Banach contraction-mapping principle in the literature (see [1-31] and others).

A new category of contractive fixed point problems was addressed by Khan et al. [1]. In this study, they introduced the notion of an altering distance function which is a control function that alters distance between two points in a metric space.

Definition 1.1. [1] A function $\phi:[0,+\infty) \rightarrow[0,+\infty)$ is called an altering distance function if the following conditions are satisfied.

(i) $\phi$ is continuous.

(ii) $\phi$ is non-decreasing.

(iii) $\phi(t)=0 \Leftrightarrow t=0$.

Khan et al. [1] proved the following result:

Theorem 1.2. [1] Let $(X, d)$ be a complete metric space, $\phi:[0,+\infty) \rightarrow[0,+\infty)$ be an altering distance function, and $T: X \rightarrow X$ be a self-mapping which satisfies the following inequality:

$$
\varphi(d(T x, T y)) \leq c \varphi(d(x, y))
$$

for all $x, y \in X$ and for some $0<c<1$. Then, $T$ has a unique fixed point.

Letting $\phi(t)=t$ in Theorem 1.2, we retrieve immediately the Banach contraction principle. 
In 1997, Alber and Guerre-Delabriere [2] introduced the concept of weak contractions in Hilbert spaces. This concept was extended to metric spaces in [3].

Definition 1.3. Let $(X, d)$ be a metric space. A mapping $T: X \rightarrow X$ is said to be weakly contractive if

$$
d(T x, T y) \leq d(x, y)-\varphi(d(x, y)), \quad \forall x, y \in X,
$$

where $\phi:[0,+\infty) \rightarrow[0,+\infty)$ is an altering distance function.

Theorem 1.4. [3] Let $(X, d)$ be a complete metric space and $T: X \rightarrow X$ be a weakly contractive map. Then, $T$ has a unique fixed point.

Weak inequalities of the above type have been used to establish fixed point results in a number of subsequent studies, some of which are noted in [4-7]. In [5], Choudhury introduced the concept of a generalized altering distance function.

Definition 1.5. [5] A function $\phi:[0,+\infty) \times[0,+\infty) \times[0,+\infty) \rightarrow[0,+\infty)$ is said to be a generalized altering distance function if the following conditions are satisfied:

(i) $\phi$ is continuous.

(ii) $\phi$ is non-decreasing in all the three variables.

(iii) $\phi(x, y, z)=0 \Leftrightarrow x=y=z=0$.

In [5], Choudhury proved the following common fixed point theorem:

Theorem 1.6. [5] Let $(X, d)$ be a complete metric space and $S, T: X \rightarrow X$ be two selfmappings such that the following inequality is satisfied:

$$
\Phi_{1}(d(S x, T y)) \leq \psi_{1}(d(x, y), d(x, S x), d(y, T y))-\psi_{2}(d(x, y), d(x, S x), d(y, T y))
$$

for all $x, y \in X$, where $\psi_{1}$ and $\psi_{2}$ are generalised altering distance functions, and $\Phi_{1}$ $(x)=\psi_{1}(x, x, x)$. Then, $S$ and $T$ have a common fixed point.

Recently, there have been so many exciting developments in the field of existence of fixed point in partially ordered sets (see [8-27] and the references cited therein). The first result in this direction was given by Turinici [27], where he extended the Banach contraction principle in partially ordered sets. Ran and Reurings [24] presented some applications of Turinici's theorem to matrix equations. The obtained result by Turinici was further extended and refined in [20-23].

In this article, we obtain coincidence point and common fixed point theorems in complete ordered metric spaces for mappings, satisfying a contractive condition which involves two generalized altering distance functions. Presented theorems are the extensions of Theorem 1.6 of Choudhury [5]. In addition, as an application, we study the existence of a common solution for a system of integral equations.

\section{Main Results}

At first, we introduce some notations and definitions that will be used later. The following definition was introduced by Jungck [28].

Definition 2.1. [28] Let $(X, d)$ be a metric space and $f, g: X \rightarrow X$. If $w=f x=g x$, for some $x \in X$, then $x$ is called a coincidence point of $f$ and $g$, and $w$ is called a point of coincidence of $f$ and $g$. The pair $\{f, g\}$ is said to be compatible if and only if $\lim _{n \rightarrow+\infty} d\left(f g x_{n}, g f x_{n}\right)=0$, whenever $\left\{x_{n}\right\}$ is a sequence in $X$ such that $\lim _{n \rightarrow+\infty} f x_{n}=\lim _{n \rightarrow+\infty} g x_{n}=t$ for some $t \in X$. 
Let $X$ be a nonempty set and $R: X \rightarrow X$ be a given mapping. For every $x \in X$, we denote by $R^{-1}(x)$ the subset of $X$ defined by

$$
R^{-1}(x):=\{u \in X \mid R u=x\} .
$$

In [19], Nashine and Samet introduced the following concept:

Definition 2.2. [19] Let $(X, \leq)$ be a partially ordered set, and $T, S, R: X \rightarrow X$ are given mappings, such that $T X \subseteq R X$ and $S X \subseteq R X$. We say that $S$ and $T$ are weakly increasing with respect to $R$ if for all $x \in X$, we have

$$
T x \preccurlyeq S y, \forall y \in R^{-1}(T x)
$$

and

$$
S x \preccurlyeq T y, \forall y \in R^{-1}(S x) .
$$

Remark 2.3. If $R: X \rightarrow X$ is the identity mapping $(R x=x$ for all $x \in X)$, then $S$ and $T$ are weakly increasing with respect to $R$ implies that $S$ and $T$ are weakly increasing mappings. It is noted that the notion of weakly increasing mappings was introduced in [9] (also see [16,29]).

Example 2.4. Let $X=[0,+\infty)$ endowed with the usual order $\leq$. Define the mappings $T, S, R: X \rightarrow X$ by

$$
T x=\left\{\begin{array}{l}
x \text { if } 0 \leq x<1 \\
0 \text { if } 1 \leq x
\end{array}, \quad S x=\left\{\begin{array}{l}
\sqrt{x} \text { if } 0 \leq x<1 \\
0 \text { if } 1 \leq x
\end{array}\right.\right.
$$

and

$$
R x= \begin{cases}x^{2} & \text { if } 0 \leq x<1 \\ 0 & \text { if } 1 \leq x\end{cases}
$$

Then, we will show that the mappings $S$ and $T$ are weakly increasing with respect to $R$.

Let $x \in X$. We distinguish the following two cases.

- First case: $x=0$ or $x \geq 1$.

(i) Let $y \in R^{-1}(T x)$, that is, $R y=T x$. By the definition of $T$, we have $T x=0$ and then $R y=0$. By the definition of $R$, we have $y=0$ or $y \geq 1$. By the definition of $S$, in both cases, we have $S y=0$. Then, $T x=0=S y$.

(ii) Let $y \in R^{-1}(S x)$, that is, $R y=S x$. By the definition of $S$, we have $S x=0$, and then $R y=0$. By the definition of $R$, we have $y=0$ or $y \geq 1$. By the definition of $T$, in both cases, we have $T y=0$. Then, $S x=0=T y$.

- Second case: $0<x<1$.

(i) Let $y \in R^{-1}(T x)$, that is, $R y=T x$. By the definition of $T$, we have $T x=x$ and then $R y=x$. By the definition of $R$, we have $R y=y^{2}$, and then $y=\sqrt{x}$. We have

$$
T x=x \leq S y=S \sqrt{x}=x^{1 / 4} \text {. }
$$


(ii) Let $y \in R^{-1}(S x)$, that is, $R y=S x$. By the definition of $S$, we have $S x=\sqrt{x}$, and then $R y=\sqrt{x}$. By the definition of $R$, we have $R y=y^{2}$, and then $y=x^{1 / 4}$. We have

$$
S x=\sqrt{x} \leq T y=T x^{1 / 4}=x^{1 / 4} .
$$

Thus, we proved that $S$ and $T$ are weakly increasing with respect to $R$.

Example 2.5. Let $X=\{1,2,3\}$ endowed with the partial order $\leq$ given by

$$
\preccurlyeq:=\{(1,1),(2,2),(3,3),(2,3),(3,1),(2,1)\} .
$$

Define the mappings $T, S, R: X \rightarrow X$ by

$$
\begin{aligned}
& T 1=T 3=1, T 2=3 ; \\
& S 1=S 2=S 3=1 ; \\
& R 1=1, R 2=R 3=2 .
\end{aligned}
$$

We will show that the mappings $S$ and $T$ are weakly increasing with respect to $R$.

Let $x, y \in X$ such that $y \in R^{-1}(T x)$. By the definition of $S$, we have $S y=1$. On the other hand, $T x \in\{1,3\}$ and $(1,1),(3,1) \in \leq$. Thus, we have $T x \leq S y$ for all $y \in R^{-1}(T x)$.

Let $x, y \in X$ such that $y \in R^{-1}(S x)$. By the definitions of $S$ and $R$, we have $R^{-1}(S x)=$ $R^{-1}(1)=\{1\}$. Then, we have $y=1$. On the other hand, $1=S x \leq T y=T 1=1$. Then, $S x$ $\leq T y$ for all $y \in R^{-1}(S x)$. Thus, we proved that $S$ and $T$ are weakly increasing with respect to $R$.

Our first result is as follows.

Theorem 2.6. Let $(X, \leq)$ be a partially ordered set, and suppose that there exists a metric $d$ on $X$ such that $(X, d)$ is a complete metric space. Let $T, S, R: X \rightarrow X$ be given mappings, satisfying for every pair $(x, y) \in X \times X$ such that $R x$ and Ry are comparable:

$$
\begin{aligned}
& \Phi_{1}(d(S x, T y)) \\
& \leq \psi_{1}(d(R x, R y), d(R x, S x), d(R y, T y))-\psi_{2}(d(R x, R y), d(R x, S x) \cdot d(R y, T y)),
\end{aligned}
$$

where $\psi_{1}$ and $\psi_{2}$ are generalized altering distance functions, and $\Phi_{1}(x)=\psi_{1}(x, x, x)$. We assume the following hypotheses:

(i) $T, S$, and $R$ are continuous.

(ii) $T X \subseteq R X, S X \subseteq R X$.

(iii) $T$ and $S$ are weakly increasing with respect to $R$.

(iv) the pairs $\{T, R\}$ and $\{S, R\}$ are compatible.

Then, $T, S$, and $R$ have a coincidence point, that is, there exists $u \in X$ such that $R u=$ $\mathrm{Tu}=\mathrm{Su}$.

Proof. Let $x_{0} \in X$ be an arbitrary point. Since $T X \subseteq R X$, there exists $x_{1} \in X$ such that $R x_{1}=T x_{0}$. Since $S X \subseteq R X$, there exists $x_{2} \in X$ such that $R x_{2}=S x_{1}$.

Continuing this process, we can construct a sequence $\left\{R x_{n}\right\}$ in $X$ defined by

$$
R x_{2 n+1}=T x_{2 n}, \quad R x_{2 n+2}=S x_{2 n+1}, \quad \forall n \in \mathbb{N} .
$$

We claim that

$$
R x_{n} \preccurlyeq R x_{n+1}, \quad \forall n \in \mathbb{N}^{*} .
$$


To this aim, we will use the increasing property with respect to $R$ for the mappings $T$ and $S$. From (2.2), we have

$$
R x_{1}=T x_{0} \preccurlyeq S y, \quad \forall y \in R^{-1}\left(T x_{0}\right) .
$$

Since $R x_{1}=T x_{0}, x_{1} \in R^{-1}\left(T x_{0}\right)$, and we get

$$
R x_{1}=T x_{0} \preccurlyeq S x_{1}=R x_{2} .
$$

Again,

$$
R x_{2}=S x_{1} \preccurlyeq T y, \quad \forall y \in R^{-1}\left(S x_{1}\right) .
$$

Since $x_{2} \in R^{-1}(S x 1)$, we get

$$
R x_{2}=S x_{1} \preccurlyeq T x_{2}=R x_{3} .
$$

Hence, by induction, (2.3) holds.

Without loss of the generality, we can assume that

$$
R x_{n} \neq R x_{n+1}, \quad \forall n \in \mathbb{N}^{*} .
$$

Now, we will prove our result on three steps.

Step I. We will prove that

$$
\lim _{n \rightarrow+\infty} d\left(R x_{n+1}, R x_{n+2}\right)=0 .
$$

Letting $x=x_{2 n+1}$ and $y=x_{2 n}$, from (2.3) and the considered contraction, we have

$$
\begin{aligned}
& \Phi_{1}\left(d\left(R x_{2 n+2}, R x_{2 n+1}\right)\right) \\
= & \Phi_{1}\left(d\left(S x_{2 n+1}, T x_{2 n}\right)\right) \\
\leq & \psi_{1}\left(d\left(R x_{2 n+1}, R x_{2 n}\right), d\left(R x_{2 n+1}, S x_{2 n+1}\right), d\left(R x_{2 n}, T x_{2 n}\right)\right) \\
& -\psi_{2}\left(d\left(R x_{2 n+1}, R x_{2 n}\right), d\left(R x_{2 n+1}, S x_{2 n+1}\right), d\left(R x_{2 n}, T x_{2 n}\right)\right) \\
= & \psi_{1}\left(d\left(R x_{2 n+1}, R x_{2 n}\right), d\left(R x_{2 n+1}, R x_{2 n+2}\right), d\left(R x_{2 n}, R x_{2 n+1}\right)\right) \\
& -\psi_{2}\left(d\left(R x_{2 n+1}, R x_{2 n}\right), d\left(R x_{2 n+1}, R x_{2 n+2}\right), d\left(R x_{2 n}, R x_{2 n+1}\right)\right) .
\end{aligned}
$$

Suppose that

$$
d\left(R x_{2 n+1}, R x_{2 n+2}\right)>d\left(R x_{2 n}, R x_{2 n+1}\right) .
$$

Using the property of the generalized altering function, this implies that

$$
\begin{aligned}
& \psi_{1}\left(d\left(R x_{2 n+1}, R x_{2 n}\right), d\left(R x_{2 n+1}, R x_{2 n+2}\right), d\left(R x_{2 n}, R x_{2 n+1}\right)\right) \\
& \leq \Phi_{1}\left(d\left(R x_{2 n+2}, R x_{2 n+1}\right)\right) .
\end{aligned}
$$

Hence, we obtain

$$
\begin{aligned}
& \Phi_{1}\left(d\left(R x_{2 n+2}, R x_{2 n+1}\right)\right) \\
& \leq \Phi_{1}\left(d\left(R x_{2 n+2}, R x_{2 n+1}\right)\right) \\
& -\psi_{2}\left(d\left(R x_{2 n+1}, R x_{2 n}\right), d\left(R x_{2 n+1}, R x_{2 n+2}\right), d\left(R x_{2 n}, R x_{2 n+1}\right)\right) .
\end{aligned}
$$

This implies that

$$
\psi_{2}\left(d\left(R x_{2 n+1}, R x_{2 n}\right), d\left(R x_{2 n+1}, R x_{2 n+2}\right), d\left(R x_{2 n}, R x_{2 n+1}\right)\right)=0
$$


and

$$
d\left(R x_{2 n+1}, R x_{2 n}\right)=0 .
$$

Hence, we obtain a contradiction with (2.4). We deduce that

$$
d\left(R x_{2 n}, R x_{2 n+1}\right) \geq d\left(R x_{2 n+1}, R x_{2 n+2}\right), \quad \forall n \in \mathbb{N}^{*} .
$$

Similarly, letting $x=x_{2 n+1}$ and $y=x_{2 n+2}$, from (2.3) and the considered contraction, we have

$$
\begin{aligned}
& \Phi_{1}\left(d\left(R x_{2 n+2}, R x_{2 n+3}\right)\right) \\
& \leq \psi_{1}\left(d\left(R x_{2 n+1}, R x_{2 n+2}\right), d\left(R x_{2 n+1}, R x_{2 n+2}\right), d\left(R x_{2 n+2}, R x_{2 n+3}\right)\right) \\
& -\psi_{2}\left(d\left(R x_{2 n+1}, R x_{2 n+2}\right), d\left(R x_{2 n+1}, R x_{2 n+2}\right), d\left(R x_{2 n+2}, R x_{2 n+3}\right)\right) .
\end{aligned}
$$

Suppose that

$$
d\left(R x_{2 n+2}, R x_{2 n+3}\right)>d\left(R x_{2 n+1}, R x_{2 n+2}\right) .
$$

Then, from (2.9) and (2.10), we obtain

$$
\begin{aligned}
& \Phi_{1}\left(d\left(R x_{2 n+2}, R x_{2 n+3}\right)\right) \\
& \leq \Phi_{1}\left(d\left(R x_{2 n+2}, R x_{2 n+3}\right)\right) \\
& -\psi_{2}\left(d\left(R x_{2 n+1}, R x_{2 n+2}\right), d\left(R x_{2 n+1}, R x_{2 n+2}\right), d\left(R x_{2 n+2}, R x_{2 n+3}\right)\right) .
\end{aligned}
$$

This implies that

$$
\psi_{2}\left(d\left(R x_{2 n+1}, R x_{2 n+2}\right), d\left(R x_{2 n+1}, R x_{2 n+2}\right), d\left(R x_{2 n+2}, R x_{2 n+3}\right)\right)=0
$$

and

$$
d\left(R x_{2 n+1}, R x_{2 n+2}\right)=0 .
$$

Hence, we obtain a contradiction with (2.4). We deduce that

$$
d\left(R x_{2 n+1}, R x_{2 n+2}\right) \geq d\left(R x_{2 n+2}, R x_{2 n+3}\right), \quad \forall n \in \mathbb{N} .
$$

Combining (2.8) and (2.11), we obtain

$$
d\left(R x_{n+1}, R x_{n+2}\right) \geq d\left(R x_{n+2}, R x_{n+3}\right), \quad \forall n \in \mathbb{N} .
$$

Hence, $\left\{d\left(R x_{n+1}, R x_{n+2}\right)\right\}$ is a decreasing sequence of positive real numbers. This implies that there exists $r \geq 0$ such that

$$
\lim _{n \rightarrow+\infty} d\left(R x_{n+1}, R x_{n+2}\right)=r .
$$

Define the function $\Phi_{2}:[0,+\infty) \rightarrow[0,+\infty)$ by

$$
\Phi_{2}(x)=\psi_{2}(x, x, x), \quad \forall x \geq 0 .
$$

From (2.6) and (2.12), we obtain

$$
\Phi_{1}\left(d\left(R x_{2 n+2}, R x_{2 n+1}\right)\right) \leq \Phi_{1}\left(d\left(R x_{2 n+1}, R x_{2 n}\right)\right)-\Phi_{2}\left(d\left(R x_{2 n+2}, R x_{2 n+1}\right)\right),
$$

which implies that

$$
\Phi_{2}\left(d\left(R x_{2 n+2}, R x_{2 n+1}\right)\right) \leq \Phi_{1}\left(d\left(R x_{2 n+1}, R x_{2 n}\right)\right)-\Phi_{1}\left(d\left(R x_{2 n+2}, R x_{2 n+1}\right)\right) .
$$


Similarly, from (2.9) and (2.12), we obtain

$$
\Phi_{1}\left(d\left(R x_{2 n+2}, R x_{2 n+3}\right)\right) \leq \Phi_{1}\left(d\left(R x_{2 n+1}, R x_{2 n+2}\right)\right)-\Phi_{2}\left(d\left(R x_{2 n+2}, R x_{2 n+3}\right)\right),
$$

which implies that

$$
\Phi_{2}\left(d\left(R x_{2 n+2}, R x_{2 n+3}\right)\right) \leq \Phi_{1}\left(d\left(R x_{2 n+1}, R x_{2 n+2}\right)\right)-\Phi_{1}\left(d\left(R x_{2 n+2}, R x_{2 n+3}\right)\right) .
$$

Now, combining (2.14) and (2.15), we obtain

$$
\Phi_{2}\left(d\left(R x_{k+2}, R x_{k+1}\right)\right) \leq \Phi_{1}\left(d\left(R x_{k+1}, R x_{k}\right)\right)-\Phi_{1}\left(d\left(R x_{k+2}, R x_{k+1}\right)\right), \quad \forall k \in \mathbb{N}^{*} .
$$

This implies that for all $n \in \mathbb{N}^{*}$, we have

$$
\begin{aligned}
\sum_{k=1}^{n} \Phi_{2}\left(d\left(R x_{k+2}, R x_{k+1}\right)\right) & \leq \sum_{k=1}^{n}\left[\Phi_{1}\left(d\left(R x_{k+1}, R x_{k}\right)\right)-\Phi_{1}\left(d\left(R x_{k+2}, R x_{k+1}\right)\right)\right] \\
& =\Phi_{1}\left(d\left(R x_{2}, R x_{1}\right)\right)-\Phi_{1}\left(d\left(R x_{n+2}, R x_{n+1}\right)\right) \\
& \leq \Phi_{1}\left(d\left(R x_{2}, R x_{1}\right)\right) .
\end{aligned}
$$

This implies that

$$
\sum_{n=1}^{+\infty} \Phi_{2}\left(d\left(R x_{k+2}, R x_{k+1}\right)\right)<\infty
$$

Hence,

$$
\lim _{n \rightarrow+\infty} \Phi_{2}\left(d\left(R x_{n+2}, R x_{n+1}\right)\right)=0 .
$$

Now, using (2.13), (2.16), and the continuity of $\Phi_{2}$, we obtain

$$
\psi_{2}(r, r, r)=\Phi_{2}(r)=0,
$$

which implies that $r=0$. Hence, (2.5) is proved.

Step II. We claim that $\left\{R x_{n}\right\}$ is a Cauchy sequence.

From (2.5), it will be sufficient to prove that $\left\{R x_{2 n}\right\}$ is a Cauchy sequence. We proceed by negation, and suppose that $\left\{R x_{2 n}\right\}$ is not a Cauchy sequence. Then, there exists $\varepsilon>0$ for which we can find two sequences of positive integers $\{m(i)\}$ and $\{n(i)\}$ such that for all positive integer $i$,

$$
n(i)>m(i)>i, d\left(R x_{2 m(i)}, R x_{2 n(i)}\right) \geq \varepsilon, d\left(R x_{2 m(i)}, R x_{2 n(i)-2}\right)<\varepsilon .
$$

From (2.17) and using the triangular inequality, we get

$$
\begin{aligned}
\varepsilon \leq & d\left(R x_{2 m(i)}, R x_{2 n(i)}\right) \\
\leq & d\left(R x_{2 m(i)}, R x_{2 n(i)-2}\right)+d\left(R x_{2 n(i)-2}, R x_{2 n(i)-1}\right) \\
& +d\left(R x_{2 n(i)-1}, R x_{2 n(i)}\right) \\
< & \varepsilon+d\left(R x_{2 n(i)-2}, R x_{2 n(i)-1}\right)+d\left(R x_{2 n(i)-1}, R x_{2 n(i)}\right) .
\end{aligned}
$$

Letting $i \rightarrow+\infty$ in the above inequality, and using (2.5), we obtain

$$
\lim _{i \rightarrow+\infty} d\left(R x_{2 m(i)}, R x_{2 n(i)}\right)=\varepsilon .
$$

Again, the triangular inequality gives us

$$
\left|d\left(R x_{2 n(i)}, R x_{2 m(i)-1}\right)-d\left(R x_{2 n(i)}, R x_{2 m(i)}\right)\right| \leq d\left(R x_{2 m(i)-1}, R x_{2 m(i)}\right) .
$$


Letting $i \rightarrow+\infty$ in the above inequality, and using (2.5) and (2.18), we get

$$
\lim _{i \rightarrow+\infty} d\left(R x_{2 n(i)}, R x_{2 m(i)-1}\right)=\varepsilon .
$$

On the other hand, we have

$$
\begin{aligned}
d\left(R x_{2 n(i)}, R x_{2 m(i)}\right) & \leq d\left(R x_{2 n(i)}, R x_{2 n(i)+1}\right)+d\left(R x_{2 n(i)+1}, R x_{2 m(i)}\right) \\
& =d\left(R x_{2 n(i)}, R x_{2 n(i)+1}\right)+d\left(T x_{2 n(i)}, S x_{2 m(i)-1}\right) .
\end{aligned}
$$

Then, from (2.5), (2.18), and the continuity of $\Phi_{1}$, and letting $i \rightarrow+\infty$ in the above inequality, we have

$$
\Phi_{1}(\varepsilon) \leq \lim _{i \rightarrow+\infty} \Phi_{1}\left(d\left(S x_{2 m(i)-1}, T x_{2 n(i)}\right)\right) .
$$

Now, using the considered contractive condition for $x=x_{2 m(i)-1}$ and $y=x_{2 n(i)}$, we have

$$
\begin{aligned}
& \Phi_{1}\left(d\left(S x_{2 m(i)-1}, T x_{2 n(i)}\right)\right) \\
& \leq \psi_{1}\left(d\left(R x_{2 m(i)-1}, R x_{2 n(i)}\right), d\left(R x_{2 m(i)-1}, R x_{2 m(i)}\right), d\left(R x_{2 n(i)}, R x_{2 n(i)+1}\right)\right) \\
& -\psi_{2}\left(d\left(R x_{2 m(i)-1}, R x_{2 n(i)}\right), d\left(R x_{2 m(i)-1}, R x_{2 m(i)}\right), d\left(R x_{2 n(i)}, R x_{2 n(i)+1}\right)\right) .
\end{aligned}
$$

Then, from (2.5), (2.19), and the continuity of $\psi_{1}$ and $\psi_{2}$, and letting $i \rightarrow+\infty$ in the above inequality, we have

$$
\lim _{i \rightarrow+\infty} \Phi_{1}\left(d\left(S x_{2 m(i)-1}, T x_{2 n(i)}\right)\right) \leq \psi_{1}(\varepsilon, 0,0)-\psi_{2}(\varepsilon, 0,0) \leq \Phi_{1}(\varepsilon)-\psi_{2}(\varepsilon, 0,0) .
$$

Now, combining (2.20) with the above inequality, we get

$$
\Phi_{1}(\varepsilon) \leq \Phi_{1}(\varepsilon)-\psi_{2}(\varepsilon, 0,0)
$$

which implies that $\psi_{2}(\varepsilon, 0,0)=0$, that is a contradiction since $\varepsilon>0$. We deduce that $\left\{R x_{n}\right\}$ is a Cauchy sequence.

Step III. Existence of a coincidence point.

Since $\left\{R x_{n}\right\}$ is a Cauchy sequence in the complete metric space $(X, d)$, there exists $u$ $\in X$ such that

$$
\lim _{n \rightarrow+\infty} R x_{n}=u
$$

From (2.21) and the continuity of $R$, we get

$$
\lim _{n \rightarrow+\infty} R\left(R x_{n}\right)=R u \text {. }
$$

By the triangular inequality, we have

$$
d(R u, T u) \leq d\left(R u, R\left(R x_{2 n+1}\right)\right)+d\left(R\left(T x_{2 n}\right), T\left(R x_{2 n}\right)\right)+d\left(T\left(R x_{2 n}\right), T u\right) .
$$

On the other hand, we have

$$
R x_{2 n} \rightarrow u, \quad T x_{2 n} \rightarrow u \quad \text { as } n \rightarrow+\infty .
$$

Since $R$ and $T$ are compatible mappings, this implies that

$$
\lim _{n \rightarrow+\infty} d\left(R\left(T x_{2 n}\right), T\left(R x_{2 n}\right)\right)=0 .
$$


Now, from the continuity of $T$ and (2.21), we have

$$
\lim _{n \rightarrow+\infty} d\left(T\left(R x_{2 n}\right), T u\right)=0 .
$$

Combining (2.22), (2.24), and (2.25), and letting $n \rightarrow+\infty$ in (2.23), we obtain

$$
d(R u, T u) \leq 0,
$$

that is,

$$
R u=T u .
$$

Again, by the triangular inequality, we have

$$
d(R u, S u) \leq d\left(R u, R\left(R x_{2 n+2}\right)\right)+d\left(R\left(S x_{2 n+1}\right), S\left(R x_{2 n+1}\right)\right)+d\left(S\left(R x_{2 n+1}\right), S u\right) .
$$

On the other hand, we have

$$
R x_{2 n+1} \rightarrow u, \quad S x_{2 n+1} \rightarrow u \quad \text { as } n \rightarrow+\infty .
$$

Since $R$ and $S$ are compatible mappings, this implies that

$$
\lim _{n \rightarrow+\infty} d\left(R\left(S x_{2 n+1}\right), S\left(R x_{2 n+1}\right)\right)=0 .
$$

Now, from the continuity of $S$ and (2.21), we have

$$
\lim _{n \rightarrow+\infty} d\left(S\left(R x_{2 n+1}\right), S u\right)=0 .
$$

Combining (2.22), (2.28), and (2.29), and letting $n \rightarrow+\infty$ in (2.27), we obtain

$$
d(R u, S u) \leq 0,
$$

that is,

$$
R u=S u .
$$

Finally, from (2.26) and (2.30), we have

$$
T u=R u=S u ，
$$

that is, $u$ is a coincidence point of $T, S$, and $R$. This completes the proof.

In the next theorem, we omit the continuity hypotheses on $T, S$, and $R$.

Definition 2.7. Let $(X, \leq, d)$ be a partially ordered metric space. We say that $X$ is regular if the following hypothesis holds: if $\left\{z_{n}\right\}$ is a non-decreasing sequence in $X$ with respect to $\leq$ such that $z_{n} \rightarrow z \in X$ as $n \rightarrow+\infty$, then $z_{n} \leq z$ for all $n \in \mathbb{N}$.

Now, our second result is the following.

Theorem 2.8. Let $(X, \leq)$ be a partially ordered set, and suppose that there exists a metric $d$ on $X$ such that $(X, d)$ is a complete metric space. Let $T, S, R: X \rightarrow X$ be given mappings satisfying for every pair $(x, y) \in X \times X$ such that $R x$ and Ry are comparable,

$$
\begin{aligned}
& \Phi_{1}(d(S x, T y)) \\
& \leq \psi_{1}(d(R x, R y), d(R x, S x), d(R y, T y))-\psi_{2}(d(R x, R y), d(R x, S x), d(R y, T y)),
\end{aligned}
$$

where $\psi_{1}$ and $\psi_{2}$ are generalized altering distance functions and $\Phi_{1}(x)=\psi_{1}(x, x, x)$. We assume the following hypotheses:

(i) $X$ is regular. 
(ii) $T$ and $S$ are weakly increasing with respect to $R$.

(iii) $R X$ is a closed subset of $(X, d)$.

(iv) $T X \subseteq R X, S X \subseteq R X$.

Then, $T, S$, and $R$ have a coincidence point.

Proof. From the proof of Theorem 2.6, we have that $\left\{R x_{n}\right\}$ is a Cauchy sequence in $(R X, d)$ which is complete, since $R X$ is a closed subspace of $(X, d)$. Hence, there exists $u=R v, v \in X$ such that

$$
\lim _{n \rightarrow+\infty} R x_{n}=u=R v .
$$

Since $\left\{R x_{n}\right\}$ is a non-decreasing sequence and $X$ is regular, it follows from (2.31) that $R x_{n} \leq R v$ for all $n \in \mathbb{N}^{*}$. Hence, we can apply the considered contractive condition. Then, for $x=v$ and $y=x_{2 n}$, we obtain

$$
\begin{aligned}
\Phi_{1}\left(d\left(S v, R x_{2 n+1}\right)\right)= & \Phi_{1}\left(d\left(S v, T x_{2 n}\right)\right) \\
\leq & \psi_{1}\left(d\left(R v, R x_{2 n}\right), d(R v, S v), d\left(R x_{2 n}, R x_{2 n+1}\right)\right) \\
& -\psi_{2}\left(d\left(R v, R x_{2 n}\right), d(R v, S v), d\left(R x_{2 n}, R x_{2 n+1}\right)\right) .
\end{aligned}
$$

Letting $n \rightarrow+\infty$ in the above inequality, and using (2.5), (2.31), and the properties of $\psi_{1}$ and $\psi_{2}$, then we have

$$
\begin{aligned}
\Phi_{1}(d(S v, R v)) & \leq \psi_{1}(0, d(R v, S v), 0)-\psi_{2}(0, d(R v, S v), 0) \\
& \leq \Phi_{1}(d(S v, R v))-\psi_{2}(0, d(R v, S v), 0) .
\end{aligned}
$$

This implies that $\psi_{2}(0, d(R v, S v), 0)=0$, which gives us that $d(R v, S v)=0$, i.e.,

$$
R v=S v .
$$

Similarly, for $x=x_{2 n+1}$ and $y=v$, we obtain

$$
\begin{aligned}
\Phi_{1}\left(d\left(R x_{2 n+2}, T v\right)\right)= & \Phi_{1}\left(d\left(S x_{2 n+1}, T v\right)\right) \\
\leq & \psi_{1}\left(d\left(R x_{2 n+2}, R v\right), d\left(R x_{2 n+1}, R x_{2 n+2}\right), d(R v, T v)\right) \\
& -\psi_{2}\left(d\left(R x_{2 n+2}, R v\right), d\left(R x_{2 n+1}, R x_{2 n+2}\right), d(R v, T v)\right) .
\end{aligned}
$$

Letting $n \rightarrow+\infty$ in the above inequality, we get

$$
\begin{aligned}
\Phi_{1}(d(R v, T v)) & \leq \psi_{1}(0,0, d(R v, T v))-\psi_{2}(0,0, d(R v, T v)) \\
& \leq \Phi_{1}(d(R v, T v))-\psi_{2}(0,0, d(R v, T v))
\end{aligned}
$$

This implies that $\psi_{2}(0,0, d(R v, T v))=0$ and then,

$$
R v=T v .
$$

Now, combining (2.32) and (2.33), we obtain

$$
R v=T v=S v .
$$

Hence, $v$ is a coincidence point of $T, S$, and $R$. This completes the proof.

Now, we present an example to illustrate the obtained result given by the previous theorem. Moreover, in this example, we will show that Theorem 1.6 of Choudhury cannot be applied.

Example 2.9. Let $X=\{4,5,6\}$ endowed with the usual metric $d(x, y)=|x-y|$ for all $x, y \in X$, and $\leq:=\{(4,4),(5,5),(6,6),(6,4)\}$. Clearly, $\leq$ is a partial order on $X$. 
Consider the mappings $T, S, R: X \rightarrow X$ defined by

$$
T=S=\left(\begin{array}{lll}
4 & 5 & 6 \\
4 & 6 & 4
\end{array}\right) \text { and } R=\left(\begin{array}{lll}
4 & 5 & 6 \\
4 & 5 & 6
\end{array}\right) \text {. }
$$

We will show that $T$ and $S$ are weakly increasing with respect to $R$. In the case under study, we have to check that $T x \leq T(T x)$ for all $x \in X$.

For $x=4$, we have

$$
T 4=4 \preccurlyeq T(T 4)=T 4=4 .
$$

For $x=5$, we have

$$
T 5=6 \preccurlyeq T(T 5)=T 6=4 .
$$

For $x=6$, we have

$$
T 6=4 \preccurlyeq T(T 6)=T 4=4 .
$$

Thus, we have proved that $T$ and $S$ are weakly increasing with respect to $R$.

Now, we will show that $(X, \leq, d)$ is regular.

Let $\left\{z_{n}\right\}$ be a non-decreasing sequence in $X$ with respect to $\leq$ such that $z_{n} \rightarrow z \in X$ as $n \rightarrow+\infty$. Then, we have $z_{n} \leq z_{n+1}$, for all $n \in \mathbb{N}$.

- If $z_{0}=4$, then $z_{0}=4 \leq z_{1}$. From the definition of $\leq$, we have $z_{1}=4$. By induction, we get $z_{n}=4$ for all $n \in \mathbb{N}$ and $z=4$. Then, $z_{n} \leq z$ for all $n \in \mathbb{N}$.

- If $z_{0}=5$, then $z_{0}=5 \leq z_{1}$. From the definition of $\leq$, we have $z_{1}=5$. By induction, we get $z_{n}=5$ for all $n \in \mathbb{N}$ and $z=5$. Then, $z_{n} \leq z$ for all $n \in \mathbb{N}$.

- If $z_{0}=6$, then $z_{0}=6 \leq z_{1}$. From the definition of $\leq$, we have $z_{1} \in\{6,4\}$. By induction, we get $z_{n} \in\{6,4\}$ for all $n \in \mathbb{N}$. Suppose that there exists $p \geq 1$ such that $z_{p}=4$. From the definition of $\leq$, we get $z_{n}=z_{p}=4$ for all $n \geq p$. Thus, we have $z=4$ and $z_{n}$ $\leq z$ for all $n \in \mathbb{N}$. Now, suppose that $z_{n}=6$ for all $n \in \mathbb{N}$. In this case, we get $z=6$, and $z_{n} \leq z$ for all $n \in \mathbb{N}$. Thus, we proved that in all the cases considered, we have $z_{n} \leq z$ for all $n \in \mathbb{N}$. Then, $(X, \leq, d)$ is regular.

Now, define the functions $\psi_{1}, \psi_{2}:[0,+\infty) \times[0,+\infty) \times[0,+\infty) \rightarrow[0,+\infty)$ by

$$
\psi_{1}(t, u, v)=\frac{1}{4}(t+u+v), \text { for all } t, u, v \geq 0
$$

and

$$
\psi_{2}(t, u, v)=\frac{1}{16}(t+u+v), \text { for all } t, u, v \geq 0 .
$$

Clearly, $\psi_{1}$ and $\psi_{2}$ are the generalized altering distance functions, and for every $x, y$ $\in X$ such that $R x \leq R y$, inequality (2.1) is satisfied.

Now, we can apply Theorem 2.8 to deduce that $T, S$, and $R$ have a coincidence point $u=4$. Note that $u$ is also a fixed point of $T$ since $S=T$, and $R$ is the identity mapping. 
On the other hand, taking $x=4$ and $y=5$, we get

$$
\begin{aligned}
& \psi_{1}(d(4,5), d(4, S 4), d(5, T 5))-\psi_{2}(d(4,5), d(4, S 4), d(5, T 5)) \\
& =\psi_{1}(1,0,1)-\psi_{2}(1,0,1) \\
& =\frac{1}{2}-\frac{1}{8}=\frac{3}{8} \\
& <\Phi_{1}(d(S 4, T 5))=\Phi_{1}(2)=\frac{3}{2} .
\end{aligned}
$$

Thus, Inequality (1.2) is not satisfied for $x=4$ and $y=5$. Then, Theorem 1.6 of Choudhury [5] cannot be applied in this case.

If $R: X \rightarrow X$ is the identity mapping, we can deduce easily the following common fixed point results.

The next result is an immediate consequence of Theorem 2.6.

Corollary 2.10. Let $(X, \leq)$ be a partially ordered set, and suppose that there exists a metric $d$ on $X$ such that $(X, d)$ is a complete metric space. Let $T, S: X \rightarrow X$ be given mappings satisfying for every pair $(x, y) \in X \times X$ such that $x$ and $y$ are comparable. Then,

$$
\Phi_{1}(d(S x, T y)) \leq \psi_{1}(d(x, y), d(x, S x), d(y, T y))-\psi_{2}(d(x, y), d(x, S x), d(y, T y)),
$$

where $\psi_{1}$ and $\psi_{2}$ are generalized altering distance functions and $\Phi_{1}(x)=\psi_{1}(x, x, x)$. We assume the following hypotheses:

(i) $T$ and $S$ are continuous.

(ii) $T$ and $S$ are weakly increasing.

Then, $T$ and $S$ have a common fixed point, that is, there exists $u \in X$ such that $u=$ $\mathrm{Tu}=\mathrm{Su}$.

The following result is an immediate consequence of Theorem 2.8.

Corollary 2.11. Let $(X, \leq)$ be a partially ordered set and suppose that there exists a metric $d$ on $X$ such that $(X, d)$ is a complete metric space. Let $T, S: X \rightarrow X$ be given mappings satisfying for every pair $(x, y) \in X \times X$ such that $x$ and $y$ are comparable. Then,

$$
\Phi_{1}(d(S x, T y)) \leq \psi_{1}(d(x, y), d(x, S x), d(y, T y))-\psi_{2}(d(x, y), d(x, S x), d(y, T y)),
$$

where $\psi_{1}$ and $\psi_{2}$ are generalised altering distance functions and $\Phi_{1}(x)=\psi_{1}(x, x, x)$. We assume the following hypotheses:

(i) $X$ is regular.

(ii) $T$ and $S$ are weakly increasing.

Then, $T$ and $S$ have a common fixed point.

A number of fixed point results may be obtained by assuming different forms for the functions $\psi_{1}$ and $\psi_{2}$. In particular, fixed point results under various contractive conditions may be derived from the above theorems. For example, if we consider

$$
\begin{aligned}
& \psi_{1}(x, y, z)=k_{1} x^{s}+k_{2} y^{s}+k_{3} z^{s}, \\
& \psi_{2}(x, y, z)=(1-k)\left[k_{1} x^{s}+k_{2} y^{s}+k_{3} z^{s}\right],
\end{aligned}
$$


where $s>0$ and $0<k=k_{1}+k_{2}+k_{3}<1$, then we obtain the following results.

The next result is an immediate consequence of Corollary 2.10.

Corollary 2.12. Let $(X, \leq)$ be a partially ordered set, and suppose that there exists a metric $d$ on $X$ such that $(X, d)$ is a complete metric space. Let $T, S: X \rightarrow X$ be given mappings satisfying for every pair $(x, y) \in X \times X$ such that $x$ and $y$ are comparable. Then,

$$
[d(S x, T y)]^{s} \leq k_{1}[d(x, y)]^{s}+k_{2}[d(x, S x)]^{s}+k_{3}[d(y, T y)]^{s},
$$

where $s>0$ and $0<k=k_{1}+k_{2}+k_{3}<1$. We assume the following hypotheses:

(i) $T$ and $S$ are continuous.

(ii) $T$ and $S$ are weakly increasing.

Then, $T$ and $S$ have a common fixed point, that is, there exists $u \in X$ such that $u=$ $\mathrm{Tu}=\mathrm{Su}$.

The next result is an immediate consequence of Corollary 2.11 .

Corollary 2.13. Let $(X, \leq)$ be a partially ordered set, and suppose that there exists a metric $d$ on $X$ such that $(X, d)$ is a complete metric space. Let $T, S: X \rightarrow X$ be given mappings satisfying for every pair $(x, y) \in X \times X$ such that $x$ and $y$ are comparable. Then,

$$
[d(S x, T y)]^{s} \leq k_{1}[d(x, y)]^{s}+k_{2}[d(x, S x)]^{s}+k_{3}[d(y, T y)]^{s},
$$

where $s>0$ and $0<k=k_{1}+k_{2}+k_{3}<1$. We assume the following hypotheses:

(i) $X$ is regular.

(ii) $T$ and $S$ are weakly increasing.

Then, $T$ and $S$ have a common fixed point.

Remark 2.14. Other fixed point results may also be obtained under specific choices of $\psi_{1}$ and $\psi_{2}$.

\section{Application}

Consider the integral equations:

$$
\begin{aligned}
& u(t)=\int_{0}^{T} K_{1}(t, s, u(s)) d s+g(t), t \in[0, T], \\
& u(t)=\int_{0}^{T} K_{2}(t, s, u(s)) d s+g(t), t \in[0, T],
\end{aligned}
$$

where $T>0$.

The purpose of this section is to give an existence theorem for common solution of (3.1) using Corollary 2.13. This application is inspired in [9].

Previously, we have considered the space $C(I)(I=[0, T])$ of continuous functions defined on $I$. Obviously, this space with the metric given by

$$
d(x, y)=\sup _{t \in I}|x(t)-y(t)|, \quad \forall x, y \in C(I),
$$


is a complete metric space. $C(I)$ can also be equipped with the partial order $\leq$ given by

$$
x, y \in C(I), \quad x \preccurlyeq y \Leftrightarrow x(t) \leq y(t), \quad \forall t \in I .
$$

Moreover, in [20], it is proved that $(C(I), \leq)$ is regular.

Now, we will prove the following result.

Theorem 3.1. Suppose that the following hypotheses hold:

(i) $K_{1}, K_{2}: I \times I \times \mathbb{R} \rightarrow \mathbb{R}$, and $g: \mathbb{R} \rightarrow \mathbb{R}$ are continuous;

(ii) for all $t, s \in I$, we have

$$
\begin{aligned}
& K_{1}(t, s, u(t)) \leq K_{2}\left(t, s, \int_{0}^{T} K_{1}(s, \tau, u(\tau)) d \tau+g(s)\right), \\
& K_{2}(t, s, u(t)) \leq K_{1}\left(t, s, \int_{0}^{T} K_{2}(s, \tau, u(\tau)) d \tau+g(s)\right) ;
\end{aligned}
$$

(iii) there exists a continuous function $p: I \times I \rightarrow \mathbb{R}_{+}$such that

$$
\left|K_{1}(t, s, x)-K_{2}(t, s, y)\right| \leq p(t, s)(x-\gamma)
$$

for all $t, s \in I$ and $x, y \in \mathbb{R}$ such that $x \geq y$;

(iv) $\sup _{t \in I} \int_{0}^{T} p(t, s) d s=\alpha<1$.

Then, the integral equations (3.1) have a solution $u^{*} \in C(I)$.

Proof. Define T, $S: C(I) \rightarrow C(I)$ by

$$
T x(t)=\int_{0}^{T} K_{1}(t, s, x(s)) d s+g(t), \quad t \in I
$$

and

$$
S x(t)=\int_{0}^{T} K_{2}(t, s, x(s)) d s+g(t), \quad t \in I .
$$

Now, we will prove that $T$ and $S$ are weakly increasing. From (ii), for all $t \in I$, we have

$$
\begin{aligned}
T x(t) & =\int_{0}^{T} K_{1}(t, s, x(s)) d s+g(t) \\
& \leq \int_{0}^{T} K_{2}\left(t, s, \int_{0}^{T} K_{1}(s, \tau, x(\tau)) d \tau+g(s)\right) d s+g(t) \\
& =\int_{0}^{T} K_{2}(t, s, T x(s)) d s+g(t) \\
& =S T x(t)
\end{aligned}
$$


Similarly,

$$
\begin{aligned}
S x(t) & =\int_{0}^{T} K_{2}(t, s, x(s)) d s+g(t) \\
& \leq \int_{0}^{T} K_{1}\left(t, s, \int_{0}^{T} K_{2}(s, \tau, x(\tau)) d \tau+g(s)\right) d s+g(t) \\
& =\int_{0}^{T} K_{1}(t, s, S x(s)) d s+g(t) \\
& =T S x(t) .
\end{aligned}
$$

Then, we have $T x \leq S T x$ and $S x \leq T S x$ for all $x \in C(I)$. This implies that $T$ and $S$ are weakly increasing.

Now, for all $x, y \in C(I)$ such that $x \leq y$, by (iii) and (iv), we have

$$
\begin{aligned}
|S x(t)-T y(t)| & \leq \int_{0}^{T}\left|K_{2}(t, s, x(s))-K_{1}(t, s, y(s))\right| d s \\
& \leq \int_{0}^{T} p(t, s)(y(s)-x(s)) d s \\
& \leq d(x, y) \int_{0}^{T} p(t, s) d s \\
& \leq \alpha d(x, y) .
\end{aligned}
$$

This implies that for all $x, y \in C(I)$ such that $x \leq y$,

$$
d(S x, T y) \leq \alpha d(x, y) .
$$

Hence, the contractive condition required by Corollary 2.13 is satisfied with $s=1, k_{1}$ $=\alpha$, and $k_{2}=k_{3}=0$.

Now, all the required hypotheses of Corollary 2.13 are satisfied. Then, there exists $u^{*}$ $\in C(I)$, a common fixed point of $T$ and $S$, that is, $u^{*}$ is a solution to (3.1).

\section{Acknowledgements}

The authors thank the referees for their valuable comments and suggestions. This work was supported by the Kyungnam University Research Fund, 2010.

\section{Author details}

${ }^{1}$ Department of Mathematics, Disha Institute of Management and Technology, Satya Vihar, Vidhansabha-Chandrakhuri Marg, Naradha, Mandir Hasaud, Raipur-492101, Chhattisgarh, India ²Département de Mathématiques, Université de Tunis, Ecole Supérieure des Sciences et Techniques de Tunis, 5, avenue Taha Hussein-Tunis, B.P. 56, Bab Menara-1008,

Tunisie ${ }^{3}$ Department of Mathematics, Kyungnam University, Masan, Kyungnam 631-701, Korea

\section{Authors' contributions}

JKK and HKN conceived the study and participated in its design and coordination. JKK suggested many good ideas that are useful for achievement this paper and made the revision. HKN and BS prepared the manuscript initially and performed all the steps of proof in this research. All authors read and approved the final manuscript.

\section{Competing interests}

The authors declare that we have no competing interests.

Received: 30 December 2010 Accepted: 21 June 2011 Published: 21 June 2011

\section{References}

1. Khan MS, Swaleh M, Sessa S: Fixed point theorems by altering distances between the points. Bull Aust Math SoC 1984, 30:1-9.

2. Alber Yal, Guerre-Delabriere S: Principles of weakly contractive maps in Hilbert spaces. Oper Theory Adv App/ 1997, 98:7-22.

3. Rhoades BE: Some theorems on weakly contractive maps. Nonlinear Anal 2001, 47:2683-2693. 
4. Abbas M, Ali Khan M: Common fixed point theorem of two mappings satisfying a generalized weak contractive condition. Int J Math Math Sci 2009, 2009:9, Article ID 131068.

5. Choudhury BS: A common unique fixed point result in metric spaces involving generalized altering distances. Math Commun 2005, 10:105-110

6. Dutta PN, Choudhury BS: A generalisation of contraction principle in metric spaces. Fixed Point Theory Appl 2008, 2008:8, Article ID 406368.

7. Zhang Q, Song Y: Fixed point theory for generalized $\varphi$-weakly contraction. Appl Math Lett 2009, 22:75-78.

8. Agarwal RP, El-Gebeily MA, O'Regan D: Generalized contractions in partially ordered metric spaces. Appl Anal 2008, 87:109-116.

9. Altun I, Simsek H: Some fixed point theorems on ordered metric spaces and application. Fixed Point Theory Appl 2010, 2010:17, Article ID 621492.

10. Amini-Harandi A, Emami H: A fixed point theorem for contraction type maps in partially ordered metric spaces and application to ordinary differential equations. Nonlinear Anal 2010, 72:2238-2242.

11. Beg I, Butt AR: Fixed point for set-valued mappings satisfying an implicit relation in partially ordered metric spaces. Nonlinear Anal 2009, 71:3699-3704.

12. Beg I, Butt AR: Fixed points for weakly compatible mappings satisfying an implicit relation in partially ordered metric spaces. Carpathian J Math 2009, 25:1-12.

13. Bhaskar TG, Lakshmikantham V: Fixed point theorems in partially ordered metric spaces and applications. Nonlinear Anal 2006, 65:1379-1393.

14. Círić N, Cakić Lj, Rajović M, Ume JS: Monotone generalized nonlinear contractions in partially ordered metric spaces. Fixed Point Theory Appl 2008, 2008:11, Article ID 131294.

15. Lakshmikantham V, Cirić Lj: Coupled fixed point theorems for nonlinear contractions in partially ordered metric spaces. Nonlinear Anal 2009, 70:4341-4349.

16. Dhage BC, O'Regan D, Agrawal RP: Common fixed point theorems for a pair of countably condensing mappings in ordered Banach spaces. J Appl Math Stoch Anal 2003, 16:243-248.

17. Harjani J, Sadarangani K: Fixed point theorems for weakly contractive mappings in partially ordered sets. Nonlinear Anal 2009, 71:3403-3410.

18. Harjani J, Sadarangani K: Generalized contractions in partially ordered metric spaces and applications to ordianry differential equations. Nonlinear Anal 2010, 72:1188-1197.

19. Nashine HK, Samet B: Fixed point results for mappings satisfying $(\psi, \varphi)$-weakly contractive condition in partially ordered metric spaces. Nonlinear Anal 2011, 74:2201-2209.

20. Nieto JJ, Rodríguez-López R: Contractive mapping theorems in partially ordered sets and applications to ordianry differential equations. Order 2005, 22:223-239.

21. Nieto JJ, Rodríguez-López R: Existence and uniqueness of fixed point in partially ordered sets and applications to ordianry differential equations. Acta Math Sinica (English Series) 2007, 23(12):2205-2212.

22. Nieto JJ, Pouso RL, Rodríguez-López R: Fixed point theorems in ordered abstract spaces. Proc Am Math Soc 2007, 135:2505-2517

23. O'Regan D, Petrusel A: Fixed point theorems for generalized contractions in ordered metric spaces. J Math Anal Appl 2008, 341:1241-1252

24. Ran $A C M$, Reurings MCB: A fixed point theorem in partially ordered sets and some applications to matrix equations. Proc Am Math Soc 2004, 132(5):1435-1443.

25. Samet B: Coupled fixed point theorems for a generalized Meir-Keeler contraction in partially ordered metric spaces. Nonlinear Anal 2010, 72:4508-4517.

26. Samet B, Yazidi H: Coupled fixed point theorems in partially ordered $\varepsilon$-chainable metric spaces. J Math Comput Sci 2010, 1(3):142-151.

27. Turinici M: Abstract comparison principles and multivariable Gronwall-Bellman inequalities. J Math Anal Appl 1986, 117:100-127.

28. Jungck G: Compatible mappings and common fixed points. Int J Math Math Sci 1986, 9:771-779.

29. Dhage BC: Condensing mappings and applications to existence theorems for common solution of differential equations. Bull Korean Math Soc 1999, 36(3):565-578.

30. Boyd DW, Wong JSW: On nonlinear contractions. Proc Am Math Soc 1969, 20:458-464.

31. Reich S: Some fixed point problems. Atti Acad Naz Linei 1974, 57:194-198.

doi:10.1186/1687-1812-2011-5

Cite this article as: Nashine et al:: Fixed point results for contractions involving generalized altering distances in ordered metric spaces. Fixed Point Theory and Applications 2011 2011:5. 